\title{
Contrasting Orthogonal and Non-orthogonal Space-Time Schemes for Perfectly-Known and Estimated MIMO Channels
}

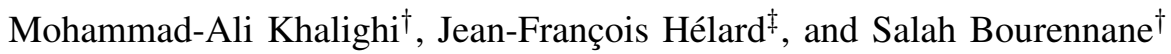 \\ ${ }^{\dagger}$ Institut Fresnel, UMR CNRS 6133, Marseille, France \\ ‡Institut d’Électronique et de Télécommunications de Rennes, UMR CNRS 6164, Rennes, France
}

\begin{abstract}
We consider the space-time coding issue in MIMO structures and compare the two potential choices of orthogonal and non-orthogonal schemes with a simple iterative detector for the latter case. We show that the choice of non-orthogonal schemes is quite rational and justified, regarding the substantial gain that we can obtain, compared to orthogonal coding. Two cases of perfectly-known channel at receiver and pilot-only-based channel estimation are studied.
\end{abstract}

\section{INTRODUCTION}

Space-time (ST) coding and decoding is an important issue in the implementation of multiple-input multiple-output (MIMO) systems [1]. MIMO structures are suitable candidates for the future cellular mobile radio systems that should provide high throughput and high quality of service. For instance, the application of MIMO systems in the fourth generation of cellular networks is considered in the European IST-4MORE project [2]. In most previous works, the design and the performance of the ST schemes have been studied in the absence of channel coding. In a practical system, however, channel coding is usually performed in order to increase the robustness against noise and interference. Now, the promised gain of one ST scheme over another may actually be too optimistic when we take into account channel coding.

Two main families of ST schemes are orthogonal block codes (OSTBC) [3], [4] and non-orthogonal schemes. Among the numerous already-proposed non-orthogonal schemes, the simplest one is spatial multiplexing or V-BLAST [5], and the most recent are linear dispersion (LD) [6] and non-vanishing codes [7]. OSTBCs can be decoded using a simple optimal detector but they generally suffer from low rate, especially for relatively large number of transmit antennas. V-BLAST schemes offer the highest rate, but cannot, in general, be employed when fewer antennas are used at receiver than at transmitter. LD codes for a desired rate are designed by maximizing the mutual information between transmitted and received signals. Codes with non-vanishing determinants, in turn, maximize rate and diversity gain such that the diversity gain is preserved for an increased signal constellation size.

The aim of this work is to compare these different solutions in view of a practical implementation of MIMO systems. To attain a desired spectral efficiency, we should set the signal constellation, the channel coding rate, and the ST scheme.
For moderate to high spectral efficiencies, it is not obvious to find the most suitable combination. If a low spectral efficiency is required, we can invest in a low-rate powerful turbo-code, using it with an OSTBC [8]. To attain high spectral efficiencies with OSTBC schemes, however, we have to increase the signal constellation size, which complicates the tasks of synchronization and detection at receiver and also results in a higher SNR required to provide a desired performance. Higher ST coding rates are offered by nonorthogonal schemes, hence, relaxing the conditions on signal constellation and channel coding. The disadvantage is that the optimal decoding is too computationally complex and practically unrealizable. One good solution would be to use a simple iterative detector for this purpose. In this way, we may approach the optimal detection performance after few iterations. Nevertheless, the detector remains more complex, as compared to the OSTBC case. We should hence investigate if this increased receiver complexity is justified, i.e., if we gain sufficiently in performance with respect to the OSTBC choice. For this study, in a first step, we assume perfect channel knowledge at receiver, and in a next step, we consider the case of estimated channel.

We present our system model and the transmission scheme in Section II. ST schemes that we consider are presented in Section III. Section IV is devoted to the decoding of ST schemes. Simulation results are presented in Section $\mathrm{V}$ to compare the performance obtained by different schemes.

\section{SYSTEM MODEL}

We consider the downlink transmission with two or four antennas at the base station (BS) and two antennas at the mobile terminal (MT). The more critical case regarding the computational complexity is in the downlink where constraints on handset power consumption and cost should be satisfied at the MT. We denote by $M_{T}$ and $M_{R}$ the number of antennas at transmitter and at receiver, respectively. We have $M_{R}=2$ and $M_{T}=2$ or $M_{T}=4$. We assume ideal multi-carrier modulation and demodulation and the absence of multi-user interference. In this way, the simplified block diagram of the transmitter is according to Fig.1. Channel coding is performed based on bit-interleaved coded modulation (BICM), using a non-recursive non-systematic convolutional (NRNSC) code $\mathcal{C}$ 


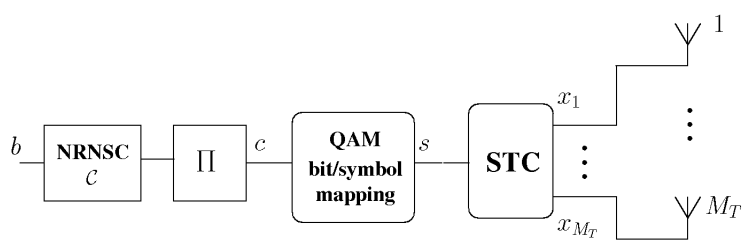

Fig. 1. Block diagram of the transmitter

and a random interleaver $\Pi$. Gray-coded QAM modulation with $B$ bits per symbol is considered. Power normalized symbols $s$ are combined according to a given ST scheme and then transmitted on $M_{T}$ antennas. We consider frequency nonselective block-fading channel model. So, each block of $N_{F}$ channel uses is described by a channel matrix $\boldsymbol{H}$ of dimension $\left(M_{R} \times M_{T}\right)$. MIMO channel fading coefficients, assumed independent and Rayleigh distributed, are considered constant in a block, changing to new independent values from one block to next. We denote by $N_{c}$ the number of independent blocks per frame. Note that for a system using ideal OFDM signaling with perfect interleaving, $N_{c}$ would correspond to the number of subcarriers and $N_{F}$ to the number of OFDM symbols per frame. Let $S$ of dimension $(Q \times 1)$ be the vector of data symbols prior to ST coding:

$$
\boldsymbol{S}=\left[s_{1}, s_{2}, \cdots, s_{Q}\right]^{t}
$$

where ${ }^{t}$ denotes transposition. By ST coding, these symbols are mapped into a matrix $\boldsymbol{X}$ of dimension $\left(M_{T} \times T\right)$ with $T$ the number of channel uses. We define the ST coding rate as $R_{S T C}=Q / T$. Let $N$ be the interleaver size. A frame of $N$ encoded bits corresponds to $\frac{N T}{B Q}$ channel uses after ST coding. In our block-fading channel model, the number of channel uses per block, $N_{F}$, is then $N_{F}=\frac{N T}{B Q N_{c}}$.

\section{CONSIDERED SPACE-TIME SCHEMES}

We are not going to present an exhaustive comparison of all already-proposed ST schemes, as this is not the aim of our work. We consider the most suitable schemes for our system, regarding ST coding rate, diversity gain, and simplicity.

\section{A. Orthogonal block codes (OSTBC)}

For $M_{T}=2$ we use the famous Alamouti code [3] with $Q=M_{T}=T=2$ and $R_{S T C}=1$, given below:

$$
\boldsymbol{X}=\left[\begin{array}{rr}
s_{1} & s_{2} \\
-s_{2}^{*} & s_{1}^{*}
\end{array}\right] \text {. }
$$

For $M_{T}=4$, we can perform Alamouti coding alternatively on one pair of antennas, while turning the other pair off. The resulting OSTBC, called time-switched Alamouti code, that we denote by $\mathrm{Sw}-\mathrm{Al}$, is given in (3) for which $Q=M_{T}=$ $T=4$ and $\boldsymbol{R}_{S T C}=1$. The normalization factor $\sqrt{2}$ ensures normalized transmit power per channel use.

$$
\boldsymbol{X}=\sqrt{2}\left[\begin{array}{rrrr}
s_{1} & s_{2} & 0 & 0 \\
-s_{2}^{*} & s_{1}^{*} & 0 & 0 \\
0 & 0 & s_{3} & s_{4} \\
0 & 0 & -s_{4}^{*} & s_{3}^{*}
\end{array}\right]
$$

We also consider the quasi-orthogonal code of Jafarkhani [9] with $Q=4, T=4, R_{S T C}=1$ and given in (4). This code provides more diversity gain than $\mathrm{Sw}-\mathrm{Al}$, but it is, of course, not orthogonal. We would like to verify whether or not without iterative detection, i.e., with a simple detector as for OSTBCs, it provides a better performance than $\mathrm{Sw}-\mathrm{Al}$.

$$
\boldsymbol{X}=\left[\begin{array}{rrrr}
s_{1} & s_{2} & s_{3} & s_{4} \\
-s_{2}^{*} & s_{1}^{*} & -s_{4}^{*} & s_{3}^{*} \\
-s_{3}^{*} & -s_{4}^{*} & s_{1}^{*} & s_{2}^{*} \\
s_{4} & -s_{3} & -s_{2} & s_{1}
\end{array}\right]
$$

\section{B. Non-orthogonal schemes}

For $M_{T}=2$, the simplest scheme is V-BLAST or spatial multiplexing, for which $Q=2, T=1$ and $R_{S T C}=2$ :

$$
\boldsymbol{X}=\left[\begin{array}{l}
s_{1} \\
s_{2}
\end{array}\right] \text {. }
$$

We also consider the LD code presented in [6] and given in (6). For this code that we denote by $\mathrm{LD}_{2 \times 2}$, we have $Q=4$, $T=2$, and $R_{S T C}=2$.

$$
\boldsymbol{X}=\frac{1}{\sqrt{2}}\left[\begin{array}{ll}
s_{1}+s_{3} & s_{2}-s_{4} \\
s_{2}+s_{4} & s_{1}-s_{3}
\end{array}\right]
$$

Another considered scheme is the optimized golden code (denoted here by GLD), presented in [7], which offers full-rate full-diversity with non-vanishing determinant. For this code, described below, we have $Q=4, T=2$, and $R_{S T C}=2$.

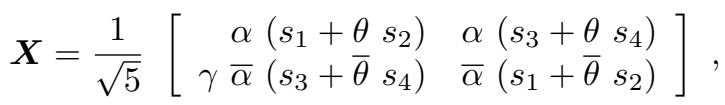

where $\theta=\frac{1+\sqrt{5}}{2}, \alpha=1+j(1-\theta), \bar{\theta}=1-\theta, \bar{\alpha}=$ $1+j(1-\bar{\theta}), \gamma=j, j=\sqrt{-1}$.

For $M_{T}=4$ and $M_{R}=2$, the V-BLAST scheme cannot be used. For this case, we use the simple double-Alamouti code (denoted by $\mathrm{D}-\mathrm{Al}$ ) with $Q=4, T=2, R_{S T C}=2$, given in (8). We also consider the optimized LD code proposed in [6] with $Q=12, T=6$ and $R_{S T C}=2$ that we denote by $\mathrm{LD}_{4 \times 2}$. We do not give its generator matrix due to space limit.

$$
\boldsymbol{X}=\left[\begin{array}{rrrr}
s_{1} & -s_{2}^{*} & s_{3} & -s_{4}^{*} \\
s_{2} & s_{1}^{*} & s_{4} & s_{3}^{*}
\end{array}\right]^{t}
$$

\section{MIMO DETECTION: ST DECODING}

Corresponding to an encoded matrix $\boldsymbol{X}$, we receive the matrix $\boldsymbol{Y}$ of dimension $\left(M_{R} \times T\right)$. We first recall the formulation of LD codes from [6] that can be used for other ST schemes as well.

\section{A. Detector formulation}

Let us denote by $\alpha_{q}$ and $\beta_{q}$ the real ( $\Re$ ) and imaginary ( $\Im$ ) parts of $s_{q}$, i.e., $s_{q}=\alpha_{q}+j \beta_{q}$. A ST scheme is described by its constructing matrices $\boldsymbol{A}_{q}$ and $\boldsymbol{B}_{q}, q=1, \cdots, Q$, all of dimension $\left(M_{T} \times T\right)$ and assumed of real-value entries, such that:

$$
\boldsymbol{X}=\sum_{q=1}^{Q}\left(\alpha_{q} \boldsymbol{A}_{q}+j \beta_{q} \boldsymbol{B}_{q}\right)
$$




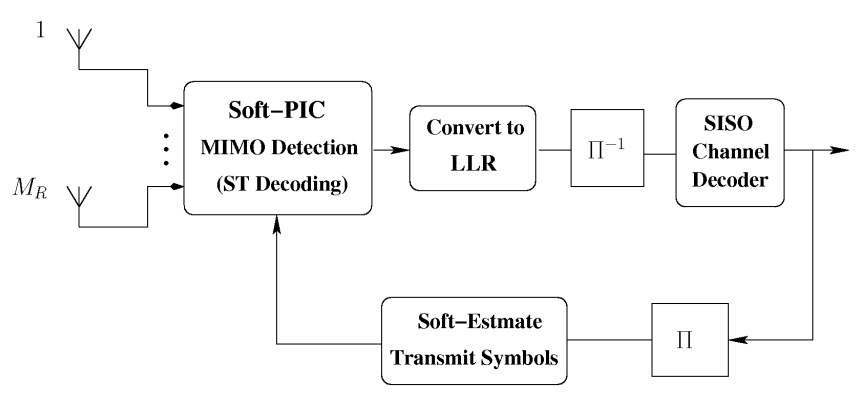

Fig. 2. Block diagram of the receiver

We separate the real and imaginary parts of the entries of $S$ and $\boldsymbol{X}$ and stack them row-wise in vectors $\mathcal{S}$ of dimension $(2 Q \times 1)$ and $\mathcal{X}$ of dimension $\left(2 M_{T} T \times 1\right)$, respectively. For instance, $\mathcal{S}=\left[\Re\left\{s_{1}\right\} \Im\left\{s_{1}\right\}, \cdots, \Re\left\{s_{Q}\right\} \Im\left\{s_{Q}\right\}\right]^{t}$.

We obtain hence, $\mathcal{X}=\mathcal{F} \mathcal{S}$, where the matrix $\mathcal{F}$ has the dimension $\left(2 M_{T} T \times 2 Q\right)$ and is obtained from the matrices $\boldsymbol{A}_{q}$ and $\boldsymbol{B}_{q}$ (see [6]). Similarly, from $\boldsymbol{Y}$ we construct the vector $\mathcal{Y}$ of dimension $\left(2 M_{R} T \times 1\right)$. Vectors $\mathcal{X}$ and $\mathcal{Y}$ are related through a matrix $\mathcal{H}$ of dimension $\left(2 M_{R} T \times 2 M_{T} T\right)$ :

$$
\mathcal{Y}=\mathcal{H} \mathcal{X}+\mathcal{N}
$$

where $\mathcal{N}$ is the vector of real AWGN of zero mean and variance $N_{0}$. Matrix $\mathcal{H}$ is composed of segments $\mathcal{H}_{i j}, i=$ $1, \cdots, M_{R}, j=1, \cdots, M_{T}$, that are block diagonal matrices of dimension $(2 T \times 2 T)$ with equal diagonal blocks $\boldsymbol{H}_{i j}$. Submatrices $\boldsymbol{H}_{i j}$ are obtained from each entry $h_{i j}$ of the initial matrix $\boldsymbol{H}$ as shown below.

$$
\boldsymbol{H}_{i j}=\left[\begin{array}{rr}
\Re\left\{h_{i j}\right\} & -\Im\left\{h_{i j}\right\} \\
\Im\left\{h_{i j}\right\} & \Re\left\{h_{i j}\right\}
\end{array}\right]
$$

Now, we can write the 'ST code and channel' input/output relationship by considering an equivalent channel matrix $\mathcal{H}_{e q}$ of dimension $\left(2 M_{R} T \times 2 Q\right)$ :

$$
\mathcal{Y}=\mathcal{H} \mathcal{F} \mathcal{S}+\mathcal{N}=\mathcal{H}_{e q} \mathcal{S}+\mathcal{N}
$$

\section{B. ST decoding}

We assume that $\mathcal{H}_{e q}$ and $N_{0}$ are known at receiver. The detection problem is now to find the transmitted data vector $\mathcal{S}$, given the vector $\mathcal{Y}$. The simplified block diagram of the receiver is shown in Fig.2. The essential detector blocks are soft-parallel interference cancellation (PIC) detector, logarithmic likelihood ratio (LLR) calculation, soft-input soft-output (SISO) channel decoder, and transmit symbols soft estimation. Detailed description of each block can be found in [10]. SISO decoding is based on Max-Log-MAP algorithm, which uses the approximation $e^{x_{1}}+e^{x_{2}} \approx \max \left(x_{1}, x_{2}\right)$. This simplifying approximation is also considered in the "Convert to LLR" part. Concerning the soft-PIC detector, at the first iteration, the detected symbols $\hat{s}_{q}$ are obtained via MMSE filtering [11]:

$$
\hat{s}_{q}=h_{q}^{\prime}\left(\mathcal{H}_{e q} \mathcal{H}_{e q}^{\prime}+\sigma_{n}^{2} \boldsymbol{I}\right)^{-1} \mathcal{Y}
$$

where $h_{q}$ of dimension $\left(2 M_{R} T \times 1\right)$ is the $q^{\text {th }}$ column of $\mathcal{H}_{e q}$. From the second iteration, we calculate soft estimates of the transmit symbols $\tilde{\mathcal{S}}$ using SISO decoder outputs and perform interference cancelling followed by zero-forcing detection:

$$
\begin{aligned}
& \hat{\mathcal{Y}}_{q}=\mathcal{Y}-\mathcal{H}_{q} \tilde{\mathcal{S}}_{q} \\
& \hat{\mathcal{S}}_{q}=\frac{1}{h_{q}^{\prime} h_{q}} h_{q}^{\prime} \hat{\mathcal{Y}}_{q}
\end{aligned}
$$

$\tilde{\mathcal{S}}_{q}$ of dimension $((2 Q-1) \times 1)$ is $\tilde{\mathcal{S}}$ with its $q^{\text {th }}$ entry removed, and $\mathcal{H}_{q}$ of dimension $\left(2 M_{R} T \times(2 Q-1)\right)$ is $\mathcal{H}_{e q}$ with its $q^{\text {th }}$ column removed. Notice that (15) is a suboptimal solution to the detection problem, which has a considerable reduced computational complexity, compared to the exact solution, given in [11], [12]. The performance loss due to this suboptimal detection would be negligible, thanks to iterative processing. Notice also that for the case of OSTBCs, the decoding is performed using (13) only.

For LLR calculation, we assume Gaussian noise plus residual interference (RI) after PIC detection [10]. Note that, as the detection is performed on blocks of $Q$ complex symbols, or in other words on blocks of $2 Q$ real symbols in our model, the RI comes in fact from $(2 Q-1)$ other real symbols in the corresponding block. This is, of course, the case only for non-orthogonal schemes. Now, in LLR calculation, we need the variance of noise plus RI [10]. This variance is calculated for the first iteration. For next iterations, however, we cannot calculate it analytically. To take into account the RI, we should hence estimate the corresponding variance in each iteration and for each one of $2 Q$ real symbols [13]. Here, to simplify the detector further, we do not estimate this variance, and we consider only the noise variance except for the first iteration. We will later see that this simplification affects considerably the performance of certain ST schemes.

\section{Simulation RESUlts}

We present a comparative study of the different ST schemes described in Section III. Performance comparison is made in terms of the bit-error-rate (BER) and for a given spectral efficiency $\eta$ (in bps/Hz). To have the same $\eta$ for different ST schemes, we accordingly set the signal constellation and the channel coding rate $R_{c}$. NRNSC code $(133,177)_{8}$ is considered where without puncturing, $R_{c}=1 / 2$. Different schemes we consider are resumed in Table I. We set $N_{c}=32$, which is the time diversity order in our simplified model, assuming perfect interleaving. SNR is considered in the form of $E_{b} / N_{0}$ and includes the receiver array gain. We denote a MIMO system with $M_{T}$ and $M_{R}$ antennas at transmitter and receiver, respectively, by $\left(M_{T} \times M_{R}\right)$. For both cases of $(2 \times 2)$ and $(4 \times 2)$ systems that we study, we set $N_{F}=24$. We first present the results for the case of perfect channel knowledge at receiver, and next take into account the channel estimation.

\section{A. Perfectly known channel at receiver}

Consider first the case of $(2 \times 2)$ MIMO system. Curves of BER versus $E_{b} / N_{0}$ for $\eta=2$ and $\eta=3 \mathrm{bps} / \mathrm{Hz}$ are shown in Fig.3 after four detector iterations for the case of nonorthogonal schemes, where almost full detector convergence is attained. We have also shown BER curves corresponding to 
TABLE I

DIFFERENT MIMO AND ST SCHEMES

\begin{tabular}{|c|c|c|c|c|}
\hline & ST scheme & $R_{S T C}$ & Modulation & $R_{c}$ \\
\hline \multirow{3}{*}{$\begin{array}{c}(2 \times 2) \\
\eta=2\end{array}$} & Alamouti & 1 & 16-QAM & $1 / 2$ \\
\hline & V-BLAST & 2 & QPSK & $1 / 2$ \\
\hline & $\mathrm{LD}_{2 \times 2}$ & 2 & QPSK & $1 / 2$ \\
\hline \multirow{3}{*}{$\begin{array}{c}(2 \times 2) \\
\eta=3\end{array}$} & Alamouti & 1 & 16-QAM & $3 / 4$ \\
\hline & V-BLAST & 2 & QPSK & $3 / 4$ \\
\hline & $\mathrm{LD}_{2 \times 2}$ & 2 & QPSK & $3 / 4$ \\
\hline \multirow{4}{*}{$\begin{array}{c}(4 \times 2) \\
\eta=3\end{array}$} & $\mathrm{Sw}-\mathrm{Al}$ & 1 & 16-QAM & $3 / 4$ \\
\hline & Jafarkhani & 1 & 16-QAM & $3 / 4$ \\
\hline & D-Al & 2 & QPSK & $3 / 4$ \\
\hline & $\mathrm{LD}_{4 \times 2}$ & 2 & QPSK & $3 / 4$ \\
\hline \multirow{4}{*}{$\begin{array}{c}(4 \times 2) \\
\eta=4\end{array}$} & Sw-Al & 1 & 64-QAM & $2 / 3$ \\
\hline & Jafarkhani & 1 & 64-QAM & $2 / 3$ \\
\hline & D-Al & 2 & 16-QAM & $1 / 2$ \\
\hline & $\mathrm{LD}_{4 \times 2}$ & 2 & 16-QAM & $1 / 2$ \\
\hline
\end{tabular}

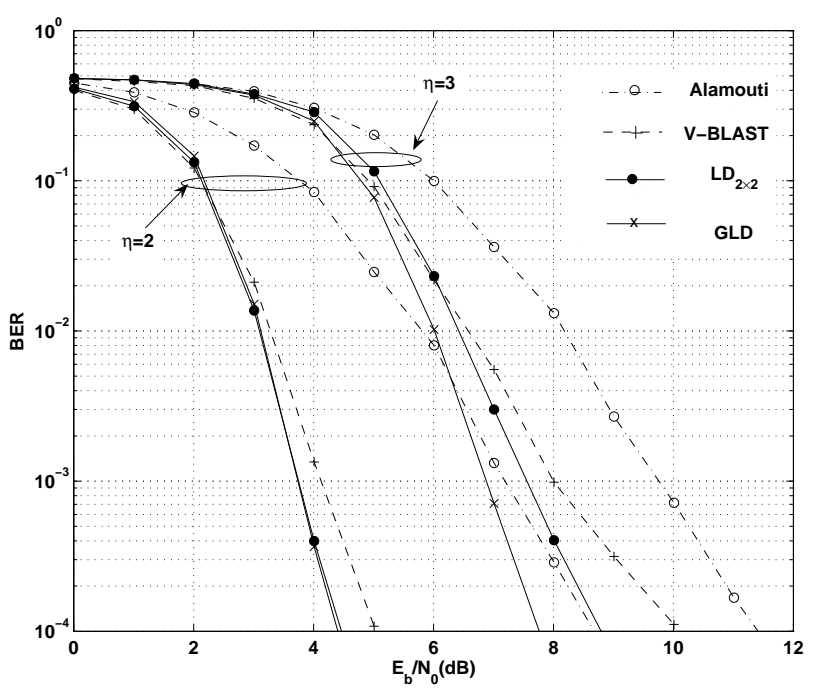

Fig. 3. $(2 \times 2)$ system, BER after 4 iterations for non-orthogonal schemes.

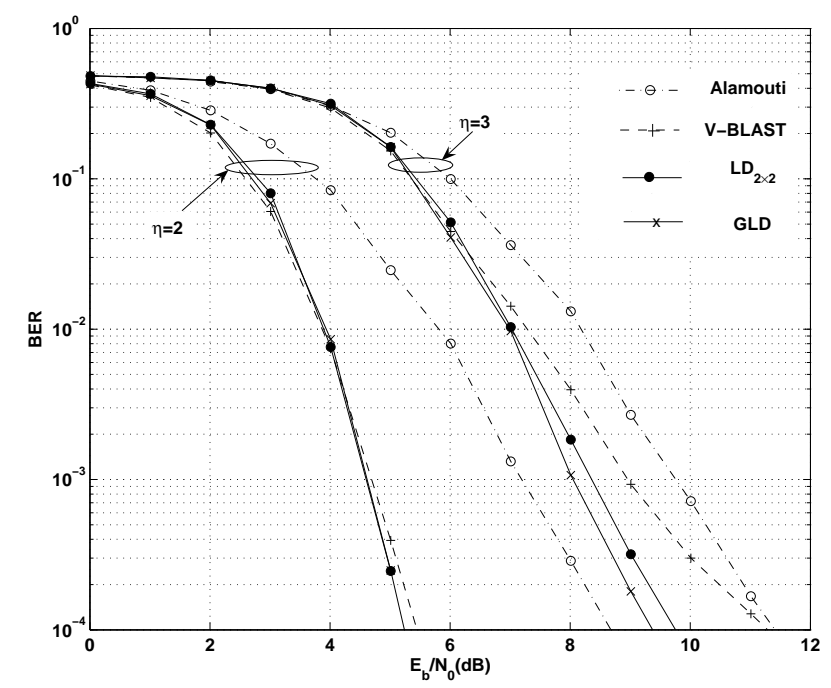

Fig. 4. $(2 \times 2)$ system, BER after 2 iterations for non-orthogonal schemes.

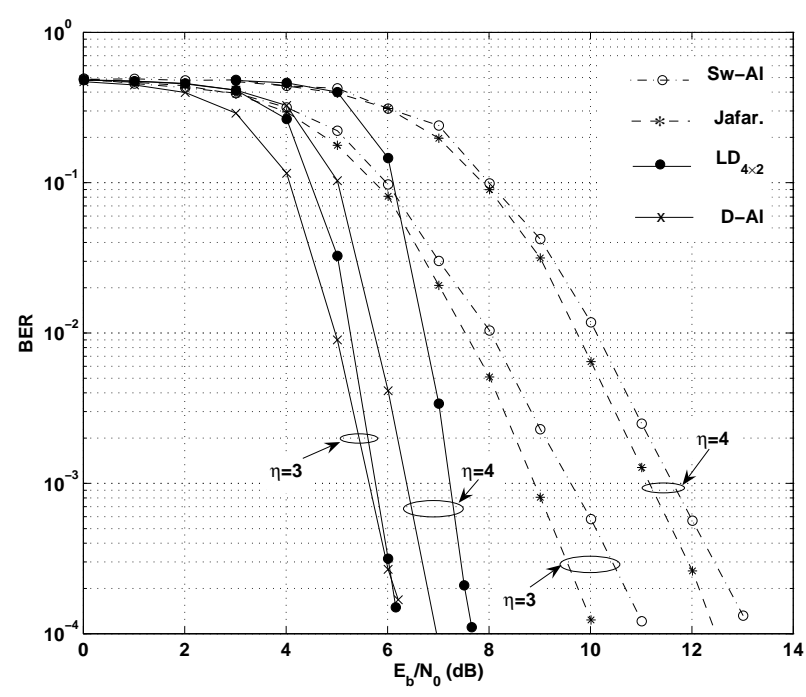

Fig. 5. $(4 \times 2)$ system, BER after 5 iterations for $\mathrm{LD}_{4 \times 2}$ and $\mathrm{D}-\mathrm{Al}$.

the second iteration in Fig.4. From Fig.3 we notice that the V-BLAST scheme undergoes an important performance degradation, as compared to $\mathrm{LD}_{2 \times 2}$ and GLD schemes, especially for $\eta=3$. We verified that this is due to the fact that we neglected RI for iterations more than one in LLR calculation (see Subsection IV-B). The present case is more critical than the case of $\eta=2$, because with $R_{c}=3 / 4$ we do not perform "enough" coding. For $\mathrm{LD}_{2 \times 2}$ and GLD schemes that offer more diversity gain, better interference rejection is done and the detector converges properly. The GLD code has the best performance as it offers full diversity. The gains obtained by using GLD after four iterations, compared to Alamouti scheme are about $4.3 \mathrm{~dB}$ and $3.7 \mathrm{~dB}$ for $\eta=2$ and $\eta=3$, respectively. The corresponding gains are respectively about $3.5 \mathrm{~dB}$ and 2.1 $\mathrm{dB}$ after two iterations of the detector. Hence, even when for the reasons of complexity and/or latency, only two iterations are to be performed, the gain in SNR compared to OSTBC choice is considerable.

Now consider the case of $(4 \times 2)$ MIMO system. Fig.5 shows performance curves for $\eta=3$ and $\eta=4$ after five iterations for non-orthogonal schemes, that corresponds to the full detector convergence. Like for $\mathrm{Sw}-\mathrm{Al}$ scheme, only one iteration is performed for the case of Jafarkhani code. We have also presented the results after two iterations for $\mathrm{LD}_{4 \times 2}$ and D-Al in Fig.6. We first notice that although the Jafarkhani code is not orthogonal, and consequently, the RI after softPIC detection by one iteration is not zero, its performance is better than $\mathrm{Sw}-\mathrm{Al}$ as it provides more diversity gain. On the other hand, we notice that D-Al has a better performance, compared to $\mathrm{LD}_{4 \times 2}$ code, especially for $\eta=4$. We again verified that this is due to sub-optimal LLR calculation. For $\mathrm{LD}_{4 \times 2}$ code, $Q$ is much larger than that for $\mathrm{D}-\mathrm{Al}$, and hence, the interference is more important. Note that another disadvantage of $\mathrm{LD}_{4 \times 2}$ scheme is that, $T$ is much larger for this code, and consequently, the detector becomes more computationally complex at the first iteration (notice the need of matrix inversion in (13)). The gain achieved by D-Al after 


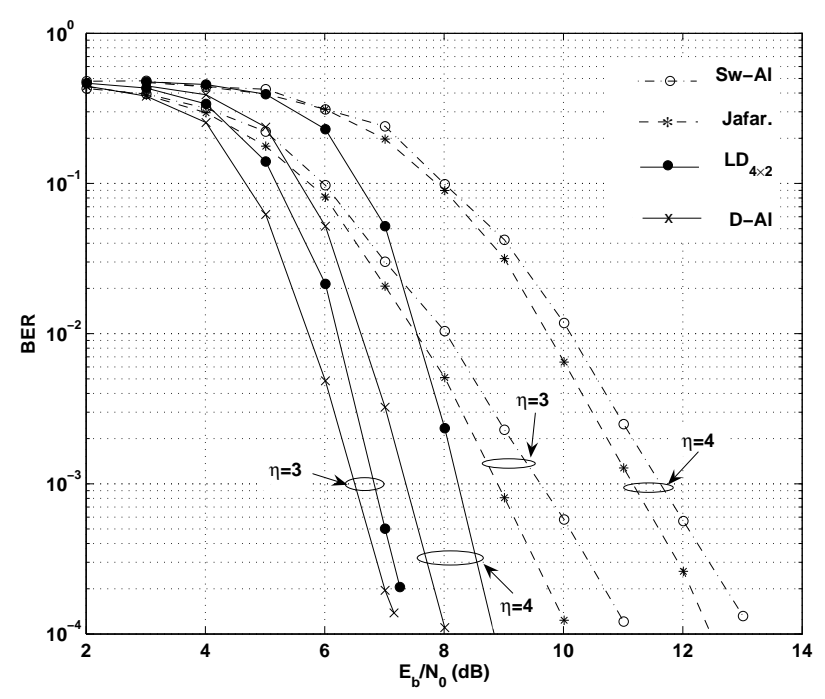

Fig. 6. $(4 \times 2)$ system, BER after 2 iterations for $\mathrm{LD}_{4 \times 2}$ and D-Al.

five iterations, compared to Jafarkhani scheme, is about 3.8 $\mathrm{dB}$ and $5.5 \mathrm{~dB}$, for $\eta=3$ and $\eta=4$, respectively. The corresponding gains are respectively about $2.8 \mathrm{~dB}$ and $4.4 \mathrm{~dB}$ after two iterations for $\mathrm{D}-\mathrm{Al}$ scheme.

\section{B. Pilot-only-based channel estimation}

It is important to study the effect of channel estimation errors on the presented results. Actually, lower-rate orthogonal schemes could be more sensitive to channel estimation errors as they have to use a larger constellation set to attain a desired spectral efficiency. Non-orthogonal schemes may in turn be more sensitive to these errors, as they affect the iterative detector convergence. Here, we consider pilot-only-based channel estimation. Using $N_{p}$ pilot bits, we devote $N_{p} /\left(B M_{T}\right)$ channel uses to the transmission of power-normalized optimal mutually orthogonal QPSK pilot sequences from $M_{T}$ antennas. We take $N_{p}$ a little greater than the required value for channel identifiability (see [10] for details). We assume that $N_{0}$ is known and pilots are used only for the estimation of $\boldsymbol{H}$. Figure 7 shows curves of SNR required to attain a BER of $10^{-4}$ for the cases of $\left(M_{T}=2, \eta=2\right)$ and $\left(M_{T}=4, \eta=4\right)$. Only GLD and D-Al are considered as appropriate non-orthogonal schemes. Note that $\eta$ does not take into account the pilots. We notice that the gain obtained by using non-orthogonal w.r.t. orthogonal schemes is still considerable and even more important for relatively small $N_{p}$ values.

\section{CONCLUSIONS}

A substantial gain can be obtained for different spectral efficiencies by using appropriate non-orthogonal schemes and a simple iterative detector at receiver, compared to orthogonal ST coding. The price paid is the increased receiver complexity that is nevertheless moderate and quite justified. As we considered the downlink transmission, we may process few iterations in order to keep the latency and the MT complexity reasonable. We noticed that even when only two iterations are performed at receiver, non-orthogonal

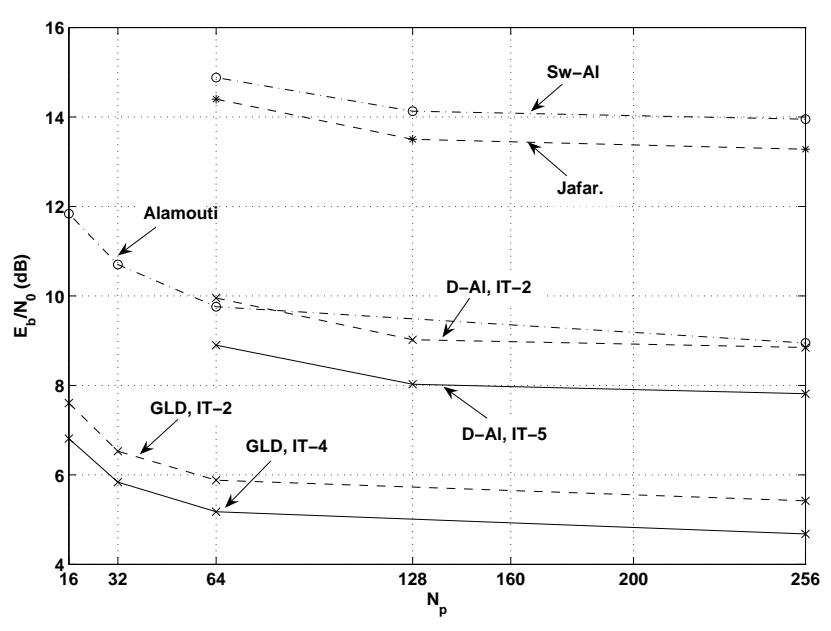

Fig. 7. SNR required to attain $\mathrm{BER}=10^{-4}, \eta=2$ for $(2 \times 2)$ and $\eta=4$ for $(4 \times 2)$ system.

schemes outperform orthogonal ones. These conclusions remain true taking into account the channel estimation errors.

\section{ACKNOWLEDGMENT}

This work was partly supported by the European IST-FP6-4MORE project (4G MC-CDMA Multiple Antenna System On Chip for Radio Enhancements), and by France-Tom R\&D / RESA / BWA.

\section{REFERENCES}

[1] B. Vucetic and J. Yuan, Space-time coding. Chichester, England: John Wiley \& Sons Ltd, 2003.

[2] "IST 4MORE project (4G MC-CDMA Multiple Antenna System On Chip for Radio Enhancements)," http://www.ist-4more.org .

[3] S. Alamouti, "A simple transmit diversity technique for wireless communications," IEEE J. Sel. Areas Commun., vol. 16, no. 8, pp. 1451-1458, Oct. 1998.

[4] V. Tarokh, H. Jafarkhani, and A. Calderbank, "Space-time block codes from orthogonal designs," IEEE Trans. Info. Th., vol. 45, no. 5, pp. 1456-1467, July 1999.

[5] G. Golden, G. Foschini, R. Valenzuela, and P. Wolniansky, "Detection algorithm and initial laboratory results using V-BLAST space-time communication architecture," Elect. Lett., vol. 35, no. 1, pp. 14-16, Jan. 1999.

[6] B. Hassibi and B. Hochwald, "High-rate codes that are linear in space and time," IEEE Trans. Info. Th., vol. 48, no. 7, pp. 1804-1824, July 2002.

[7] J.-C. Belfiore, G. Rekaya, and E. Viterbo, "The golden code: a $2 \times 2$ full-rate space-time code with nonvanishing determinants," IEEE Trans. Info. Th., vol. 51, no. 4, pp. 1432-1436, Apr. 2005.

[8] V. L. Nir, J.-M. Auffray, M. Hélard, J.-F. Hélard, and R. L. Gouable, "Combination of space-time block coding with MC-CDMA technique for MIMO systems with two, three and four transmit antennas," IST Mobile Commun. Summit Conf., June 2003.

[9] H. Jafarkhani, "A quasi-orthogonal space-time block code," IEEE Trans. Commun., vol. 49, no. 1, pp. 1-4, Jan. 2001.

[10] M. A. Khalighi, J. Boutros, and J.-F. Hélard, "Data-aided channel estimation for Turbo-PIC MIMO detectors," IEEE Commun. Lett., vol. 10, no. 5, pp. 350-352, May 2006.

[11] M. Sellathurai and S. Haykin, "Turbo-BLAST for wireless communications: theory and experiments," IEEE Trans. Sig. Proc., vol. 50, no. 10, pp. 2538- 2546, Oct. 2002.

[12] M. Witzke, S. Bäro, F. Schreckenbach, and J. Hagenauer, "Iterative detection of MIMO signals with linear detectors," 36th Asilomar Conf. Sig., Syst. \& Comp., vol. 1, pp. 289-293, Nov. 2002, Pacific Grove, CA.

[13] M. A. Khalighi and J.-F. Hélard, "Should MIMO orthogonal space-time coding be preferred to non-orthogonal coding with iterative detection?" ISSPIT Symp., pp. 340-345, Dec. 2005, Athens, Greece. 